\title{
Absolute Parallelism in The Bainchi-Type IX Taub Solution
}

\section{Murat KORUNUR ${ }^{1}$}

ABSTRACT: In this work, we study one of the famous gravitation problems in an alternative point of view which is known as the absolute parallelism. Considering Taub solution of the Bainchi-Type IX space-time and the Hamiltonian approach technique of absolute parallelism, we obtain corresponding angular momentum, total energy and momentum distributions. It is seen that our main results agree with those ones published in the literature by other author.

Keywords: Angular momentum, energy, teleparallel gravity

\section{Bianchi-Tipi IX Taub Çözümünde Mutlak Paralelizm}

ÖZET: Bu çalışmada, mutlak paralellizm olarak bilinen alternatif bir bakış açısıyla ünlü kütleçekim problemlerinden birini inceliyoruz. Bainchi-Type IX uzay-zamanın Taub çözümü ve mutlak paralellizmde Hamilton yaklaşımı tekniği göz önüne alındığında, bu duruma karşılık gelen açısal momentum, toplam enerji ve momentum dağılımları elde edilmektedir. Temel sonuçlarımızın diğer bir yazar tarafından literatürde yayınlanan sonuçlarla uyumlu olduğu görülmektedir.

Anahtar Kelimeler: Açısal Momentum, enerji, mutlak paralelizm

Murat KORUNUR (0000-0002-8311-9079), Atatürk Üniversitesi, Ziraat Fakültesi, Zootekni Bölümü, Erzurum, Türkiye

Munzur Üniversitesi, Tunceli Meslek Yüksekokulu, Elektrik ve Enerji, Tunceli, Türkiye

Sorumlu yazar/Corresponding Author: Murat KORUNUR, muratkorunur@yahoo.com 


\section{INTRODUCTION}

The energy-momentum localization is one of the interesting and unsolved phenomenons both in the general relativity and the absolute parallelism. The first attempt was done by Einstein himself (Einstein, 1915). Thereafter many prescriptions have been used and improved to solve and understand this puzzle (Tolman, 1934; Papapetrou, 1948; Landau and Liftshitz, 1951; Bergmann and Thompson, 1953; Möller, 1958; Weinberg, 1972; Qadir and Sharif, 1992, Abedi and Salti, 2015). Using the absolute parallelism description of Einstein's general relativity, Maluf (Maluf, 1994) established Hamilton approach. Later, Maluf and his collaborators (Maluf and Rocha-Neto, 1999, 2001) found the localized energy density of rotating black holes and null surfaces in the absolute parallelism. Next, in the context of Kerr black hole, the gravitational energy, momentum and angular momentum distributions were calculated (Maluf et al., 2002). Making use of the Banados-Teitelboim-Zanelli (BTZ) black hole and the Hamilton approach of three dimensional absolute parallelism, total angular momentum distribution associated with the spacetime model is found (Sousa and Pareira, 2006). Recently, many scientist have interested in this interesting approach to discuss the four-momentum localization problem in various spacetime models (Nashed, 2008, 2010; Sousas et al., 2010; Maluf and Ulhoa, 2009; Sharif and Taj, 2010; Ulhoa and Rocha, 2013; Maluf, 2013; Saltı and Açikgöz, 2013; Rocha Neto and Maluf, 2014).

Here, we use the following representations: the space-time indices defined by the Greek alphabet () while the tangent space indices described by the Latin alphabet $(\alpha, \beta, \psi) .((a),(b),(c)$...) All indices run from to . Time and space indices are denoted according to where $\mu=0, \hat{\mu}$ where $\hat{\mu}=1,2,3$.

\section{MATERIAL AND METHODS}

The basic geometry of absolute parallelism is the tetrad field $h_{\mu}^{(a)}$ and the absolute parallelism is defined on Weitzenböck space-time (Weitzenböck, 1923):

$$
\Gamma_{\mu \nu}^{\sigma}=h_{(a)}^{\sigma} \partial_{\mu} h_{v}^{(a)}
$$

where $g_{\mu \nu}=\eta_{(a)(b)} h_{\mu}^{(a)} h_{v}^{(b)}$ with $\eta_{(a)(b)}$ is the Minkowski metric. The torsion tensor can be defined in the absolute parallelism as given below (Hehl, 1980):

$$
T_{\mu \nu}^{(a)}=\partial_{\mu} h_{v}^{(a)}-\partial_{\nu} h_{\mu}^{(a)}
$$

The Lagrangian density in absolute parallelism (Maluf, 1994) is written as

$$
\mathcal{L}=-\kappa h\left[\frac{1}{4} T^{(a)(b)(c)} T_{(a)(b)(c)}+\frac{1}{2} T^{(a)(b)(c)} T_{(b)(a)(c)}-T^{(a)} T_{(a)}\right]-\mathcal{L}_{M} .
$$

Here $h=\operatorname{det}\left(h_{\mu}^{(a)}\right), \kappa=\frac{1}{16 \pi}$ and the trace of torsion tensor is defined by $T_{(a)}=T_{(b)(a)}^{b}$.

Variation of the Equation 3. with respect to tetrad components $\left(h^{(a) \mu}\right)$ gives the corresponding field equations

$$
\begin{aligned}
& \frac{\delta \mathcal{L}_{M}}{\delta h^{(a) \mu}}=h T_{(a) \mu}=4 \kappa h_{(a) \sigma} h^{(b) \mu} \partial_{\nu}\left(h \Sigma^{(b) \sigma v}\right) \\
& -4 \kappa h\left(\Sigma_{(a)}^{(b) v} T_{(b) v \mu}-\frac{1}{4} h_{(a) \mu} T_{(k)(l)(m)} \Sigma^{(k)(l)(m)}\right) .
\end{aligned}
$$


Here, the $\Sigma^{(a)(b)(c)}$ is an antisymmetric tensor according to the last two indices;

$$
\begin{aligned}
& \Sigma^{(a)(b)(c)}=\frac{1}{4}\left[T^{(a)(b)(c)}+T^{(b)(a)(c)}-T^{(c)(a)(b)}\right] \\
& +\frac{1}{2}\left[\eta^{(a)(c)} T^{(b)}-\eta^{(a)(b)} T^{(c)}\right] .
\end{aligned}
$$

Next, the total Hamiltonian density is given by (Maluf and Rocha-Neto, 2001)

$$
\mathcal{H}=h_{(a)(0)} \mathcal{F}^{(a)}+\phi_{(a)(b)} \Gamma^{(a)(b)}+\psi_{(b)} \Gamma^{(b)}+\partial_{\widehat{\lambda}}\left(h_{(a) 0} \Pi^{(a) \widehat{\lambda}}\right)
$$

where $\Gamma^{(a)(b)}$ and $\Gamma^{(b)}$ are constrains, and

$$
\begin{aligned}
& \phi_{(a)(b)}=\frac{1}{2}\left[T_{(a) 0(b)}+T_{(b) 0(a)}\right] \\
& \psi_{(b)}=T_{(00(b)}
\end{aligned}
$$

are known as the Lagrangian multipliers. On the other hand, the total Lagrangian density is also given by

$$
\mathcal{F}^{(a)}=-\partial_{\widehat{\lambda}} \Pi^{(a)} \widehat{\hat{\lambda}}+\mathcal{H}^{(a)},
$$

with

$$
\Pi^{(a) \widehat{\lambda}}=-4 \kappa h \Sigma^{(a) 0 \widehat{\lambda}}
$$

where $\mathcal{H}^{(a)}$ denotes a vector constraint which is obtained from $\mathcal{F}^{(a)}=0$.

Furthermore, for an arbitrary space volume, the energy-momentum density is defined by $-\partial_{\widehat{\lambda}} \Pi^{(a) \widehat{\lambda}}$ while the total energy-momentum is given as (Maluf and Rocha-Neto, 2001)

$$
P^{(a)}=-\int_{V}\left[\partial_{\widehat{\lambda}} \Pi^{(a) \hat{\lambda}}\right] d^{3} x
$$

In addition to this, the four-angular momentum of gravitational fields is defined by (Maluf et al., 2006)

$$
L^{(a)(b)}=-\int_{V} M^{(a)(b)} d^{3} x
$$

where $M^{(a)(b)}$ represents angular momentum density and it is given by

$$
M^{(a)(b)}=2 \kappa \partial_{\widehat{\lambda}}\left[h\left(h^{(a) \widehat{\lambda}} h^{(b) 0}-h^{(b) \hat{\lambda}} h^{(a) 0}\right)\right] .
$$


In the present work, we consider the Hamiltonian formulation in the absolute parallelism to obtain angular momentum, total energy and momentum distributions associated with the Taub type spacetime model. In the next section, the total angular momentum and four-momentum densities are calculated. We also plot these quantities versus time coordinate to discuss their evolutionary behaviors.
The last section is devoted to the summary and final remarks.

\section{RESULTS AND DISCUSSION}

In the holonomic coordinate system the special case of homogenous isotropic Bianchi Type IX model is given by (Taub, 1954):

$$
\begin{aligned}
& d s^{2}=F(t) d t^{2}-Y(t) d x^{2}-\left[Y(t) \sin ^{2}(x)+H(t) \cos ^{2}(x)\right] d y^{2} \\
& -H(t) d z^{2}-2 H(t) \cos (x) d y d z
\end{aligned}
$$

where

$$
\begin{aligned}
& Y(t)=\frac{\omega \cosh (\omega t+\xi)}{4 \cosh ^{2}\left(\frac{\omega t+\sigma}{2}\right)}, \\
& H(t)=\frac{\omega}{\cosh (\omega t+\xi)}, \\
& F(t)=Y^{2}(t) H(t) .
\end{aligned}
$$

Here $\omega, \xi, \sigma$ show there constants, $x, y$ and $z$ ranges within:

$$
0 \leq x \leq \pi ; \quad 0 \leq y \leq 4 \pi ; \quad 0 \leq z \leq 2 \pi
$$

Now, the metric tensor can be written as

$$
g_{\mu \nu}=\left(\begin{array}{llll}
F & 0 & 0 & 0 \\
0 & -Y & 0 & 0 \\
0 & 0 & -\left[Y \sin ^{2}(x)+H \cos ^{2}(x)\right] & -H \cos (x) \\
0 & 0 & -H \cos (x) & -H
\end{array}\right)
$$

In order to obtain tetrad fields, we use the Equations 13., 14., 15., the metric tensor 17., and the relationship $g_{\mu \nu}=h_{\mu}^{(a)} h_{(a) v}$. Hence, one can easily obtain the following components

$$
\begin{aligned}
& h_{\mu}^{(0)}=\left(\frac{\omega^{3 / 2} \cosh ^{1 / 2}(\omega t+\xi)}{4 \cosh ^{2}\left(\frac{\omega t+\sigma}{2}\right)}, 0,0,0\right), \\
& h_{\mu}^{(1)}=\left(0, \frac{\omega^{1 / 2} \cosh ^{1 / 2}(\omega t+\xi)}{2 \cosh \left(\frac{\omega t+\sigma}{2}\right)}, 0,0\right),
\end{aligned}
$$




$$
\begin{aligned}
& h_{\mu}^{(2)}=\left(0,0, \frac{\omega^{1 / 2} \sin (x) \cosh ^{1 / 2}(\omega t+\xi)}{2 \cosh \left(\frac{\omega t+\sigma}{2}\right)}, 0\right) \\
& h_{\mu}^{(3)}=\left(0,0,-\frac{\cos (x)}{\omega^{1 / 2} \cosh ^{1 / 2}(\omega t+\xi)}, \frac{\omega^{1 / 2}}{\cosh ^{1 / 2}(\omega t+\xi)}\right) .
\end{aligned}
$$

Next, considering $h_{(a)}^{\mu}=g^{\mu \nu} h_{(a) v}$, the inverse tetrad components are found as in the following forms

$$
\begin{aligned}
& h_{(0)}^{\mu}=\left(\frac{4 \cosh ^{2}\left(\frac{\omega t+\sigma}{2}\right)}{\omega^{3 / 2} \cosh ^{1 / 2}(\omega t+\xi)}, 0,0,0\right), \\
& h_{(1)}^{\mu}=\left(0, \frac{2 \cosh \left(\frac{\omega t+\sigma}{2}\right)}{\omega^{1 / 2} \cosh ^{1 / 2}(\omega t+\xi)}, 0,0\right), \\
& h_{(2)}^{\mu}=\left(0,0, \frac{2 \cosh \left(\frac{\omega t+\sigma}{2}\right)}{\omega^{1 / 2} \sin (x) \cosh { }^{1 / 2}(\omega t+\xi)},-\frac{2 \cot (x) \cosh \left(\frac{\omega t+\sigma}{2}\right)}{\omega^{1 / 2} \cosh ^{1 / 2}(\omega t+\xi)}\right), \\
& h_{(3)}^{\mu}=\left(0,0,0, \frac{\omega^{1 / 2}}{\cosh ^{1 / 2}(\omega t+\xi)}\right) .
\end{aligned}
$$

It is concluded that tetrad fields satisfy $h_{(0)}^{i}=0$. So, the three velocity conditions is ensured and the frame is static.

Thus, the non-vanishing components of torsion tensor are calculated as

$$
\begin{aligned}
& T^{(1)(0)(1)}=-T^{(1)(1)(0)}=\frac{2 \cosh \left(\frac{\omega t+\sigma}{2}\right) \sinh \left(\frac{\omega t}{2}+\xi-\frac{\sigma}{2}\right)}{\omega^{1 / 2} \cosh ^{3 / 2}(\omega t+\xi)}, \\
& T^{(2)(0)(2)}=-T^{(2)(2)(0)}=\frac{2 \cosh \left(\frac{\omega t+\sigma}{2}\right) \sinh \left(\frac{\omega t}{2}+\xi-\frac{\sigma}{2}\right)}{\omega^{1 / 2} \cosh ^{3 / 2}(\omega t+\xi)}, \\
& T^{(2)(1)(2)}=-T^{(2)(2)(1)}=\frac{2 \cosh \left(\frac{\omega t+\sigma}{2}\right) \cot (x)}{\omega^{1 / 2} \cosh ^{1 / 2}(\omega t+\xi)} \\
& T^{(3)(0)(3)}=-T^{(3)(3)(0)}=-\frac{2 \cosh ^{2}\left(\frac{\omega t+\sigma}{2}\right) \sinh (\omega t+\xi)}{\omega^{1 / 2} \cosh ^{3 / 2}(\omega t+\xi)}, \\
& T^{(3)(1)(2)}=-T^{(3)(2)(1)}=\frac{4 \cosh ^{2}\left(\frac{\omega t+\sigma}{2}\right)}{\omega^{3 / 2} \cosh ^{3 / 2}(\omega t+\xi)}
\end{aligned}
$$


while the surviving components of $T^{i}$ is found as given below

$$
\begin{aligned}
& T^{(0)}=\frac{\cosh \left(\frac{\omega t+\sigma}{2}\right)\left[\sinh \left(\frac{3 \omega t+2 \xi+\sigma}{2}\right)-3 \sinh \left(\frac{\omega t}{2}+\xi-\frac{\sigma}{2}\right)\right]}{\omega^{1 / 2} \cosh ^{3 / 2}(\omega t+\xi)}, \\
& T^{(1)}=\frac{2 \cosh \left(\frac{\omega t+\sigma}{2}\right) \cot (x)}{\omega^{1 / 2} \cosh ^{1 / 2}(\omega t+\xi)} .
\end{aligned}
$$

Additionally, the non-vanishing components of $\Sigma^{(a)(b)(c)}$ become:

$$
\begin{aligned}
& \Sigma^{(0)(0)(1)}=-\Sigma^{(0)(1)(0)}=\Sigma^{(3)(1)(3)}=-\Sigma^{(3)(3)(1)}=-\frac{\cosh \left[\frac{1}{2}(\omega t+\sigma)\right] \cot (x)}{\omega^{1 / 2} \cosh ^{1 / 2}(\omega t+\xi)} \\
& \Sigma^{(1)(0)(1)}=-\Sigma^{(1)(1)(0)}=\Sigma^{(2)(0)(2)}=-\Sigma^{(2)(2)(0)}=\frac{\cosh \left[\frac{1}{2}(\omega t+\sigma)\right]}{2 \omega^{1 / 2} \cosh ^{3 / 2}(\omega t+\xi)^{\prime}} \\
& \times\left[5 \sinh \left(\frac{\omega t}{2}+\xi-\frac{\sigma}{2}\right)-\sinh \left(\frac{3 \omega t}{2}+\xi+\frac{\sigma}{2}\right)\right] \\
& \Sigma^{(1)(2)(3)}=-\Sigma^{(1)(3)(2)}=-\Sigma^{(2)(1)(3)}=\Sigma^{(2)(3)(1)} \\
& =-\Sigma^{(3)(1)(2)}=\Sigma^{(3)(2)(1)}=-\frac{\cosh ^{2}\left[\frac{1}{2}(\omega t+\sigma)\right]}{\omega^{3 / 2} \cosh ^{3 / 2}(\omega t+\xi)^{\prime}} \\
& \Sigma^{(3)(0)(3)}=-\Sigma^{(3)(3)(0)}=-\frac{\sinh ^{1 / 2}(\omega t+\sigma)}{\omega^{1 / 2} \cosh ^{1 / 2}(\omega t+\xi)}
\end{aligned}
$$

Therefore, one can easily get the $\Pi^{(a)(b)}$ components

$$
\begin{aligned}
& \Pi^{(0)(1)}=\frac{\omega \cos x}{16 \pi \cosh (\omega t+\sigma)} \\
& \Pi^{(1)(1)}=\Pi^{(2)(2)}=\frac{\omega\left[\sinh \left(\frac{3 \omega t}{2}+\xi+\frac{\sigma}{2}\right)-5 \sinh \left(\frac{\omega t}{2}+\xi-\frac{\sigma}{2}\right)\right]}{32 \pi \cosh (\omega t+\xi) \cosh \left(\frac{\omega t}{2}+\frac{\sigma}{2}\right)} \sin (x), \\
& \Pi^{(3)(3)}=\frac{\omega}{8 \pi} \tanh \left(\frac{\omega t}{2}+\frac{\xi}{2}\right) \sin (x) .
\end{aligned}
$$

Now, making use of the relation

$$
\Pi^{(a) \mu}=h_{(b)}^{\mu} \Pi^{(a)(b)},
$$

it can be obtained that

$$
\begin{aligned}
& \Pi^{(0) 1}=\frac{\omega^{1 / 2} \cos (x)}{8 \pi \cosh ^{1 / 2}(\omega t+\xi)} \\
& \Pi^{(1) 1}=\frac{\omega^{1 / 2}\left[\sinh \left(\frac{3 \omega t}{2}+\xi+\frac{\sigma}{2}\right)-5 \sinh \left(\frac{\omega t}{2}+\xi-\frac{\sigma}{2}\right)\right]}{16 \pi \cosh ^{3 / 2}(\omega t+\xi)} \sin (x),
\end{aligned}
$$




$$
\begin{aligned}
& \Pi^{(2) 2}=\frac{\omega^{1 / 2}\left[\sinh \left(\frac{3 \omega t}{2}+\xi+\frac{\sigma}{2}\right)-5 \sinh \left(\frac{\omega t}{2}+\xi-\frac{\sigma}{2}\right)\right]}{16 \pi \cosh ^{3 / 2}(\omega t+\xi)}, \\
& \Pi^{(2) 3}=\frac{\omega^{-1 / 2}\left[\sinh \left(\frac{3 \omega t}{2}+\xi+\frac{\sigma}{2}\right)-5 \sinh \left(\frac{\omega t}{2}+\xi-\frac{\sigma}{2}\right)\right]}{16 \pi \cosh ^{3 / 2}(\omega t+\xi)} \cos (x), \\
& \Pi^{(3) 3}=\frac{\omega^{1 / 2}}{8 \pi} \cosh ^{1 / 2}(\omega t+\xi) \tanh \left(\frac{\omega t}{2}+\frac{\sigma}{2}\right) \sin (x) .
\end{aligned}
$$

When we use the results given in Equation 19. with the Equation 11., it can be calculated that the angular momentum density becomes

$$
M^{(0)(1)}=-M^{(1)(0)}=\frac{\kappa \omega \cos (x)}{\cosh \left(\frac{\omega t+\sigma}{2}\right)} .
$$

From this point of view, for the total angular momentum, we obtain

$$
L^{a b}=0 .
$$

In addition to this, the total energy associated with the Taub type space-time solution is found as

$$
E=-\int_{V} d^{3} x \partial_{i} \Pi^{(0) i}=2 \pi \omega^{1 / 2} \operatorname{sech}^{1 / 2}(\omega t+\xi) .
$$

Besides, the corresponding momentum becomes

$$
P^{i}=0 \text {. }
$$

\section{CONCLUSION}

In this work, we mainly consider the absolute parallelism instead of Einstein's theory of general relativity. Making use of the Hamiltonian approach, we calculate the total angular momentum, energy and the

$$
E=2 \pi \omega^{1 / 2} \operatorname{sech}^{1 / 2}(\omega t+\xi)
$$

which is similar that one published already in the literature previously by Halpern (Halpern, 2006). Halpern performed corresponding calculations by using the Einstein, Papapetrou, Landau-Lifshitz and Möller momentum for the Taub solution of Bainchi-Type IX Spacetime.

For the total energy distribution, the localized relation is found as:

prescriptions in the general theory of relativity. That's why, we have shown also that two different points of view proposed to discuss the nature of gravitation are agree with each other. 


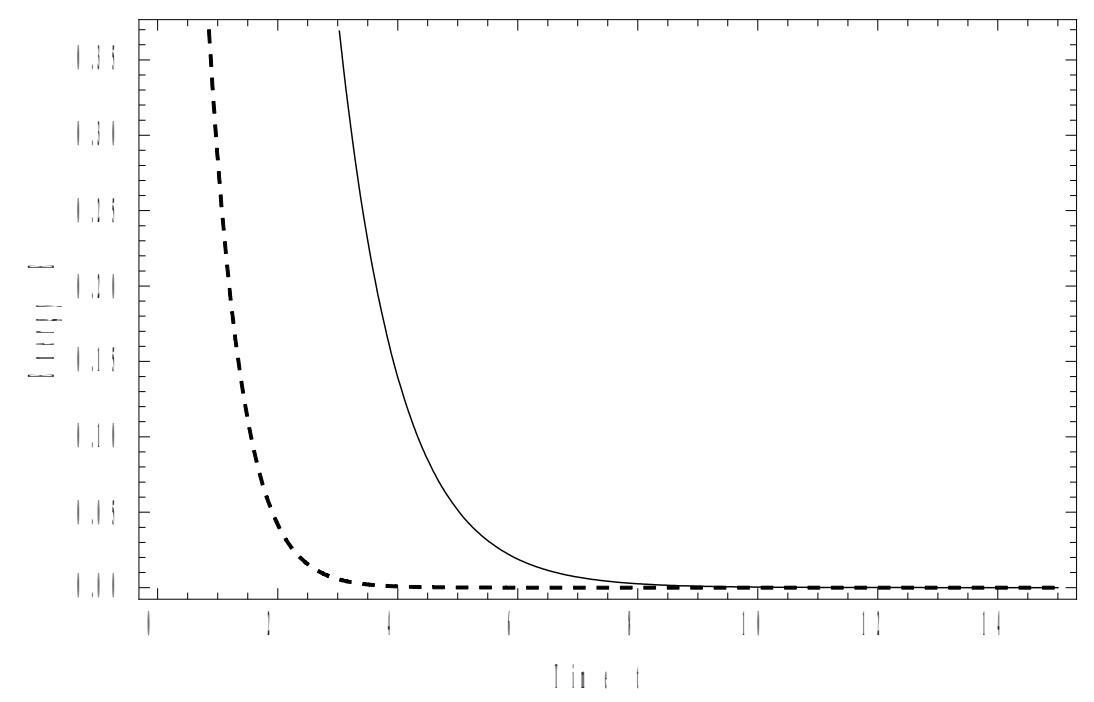

Figure 1: The total energy distribution versus time. Black line corresponds our results and dashed line corresponds Halpern results. Here, we have taken the constants as $\omega=2, \xi=1, \sigma=1$

Figure 1 shows us both Halpern and our results obtained for the total energy according to the time coordinate. It is obviously seen that the total energy

\section{REFERENCES}

Abedi H, Salti M, 2015. Multiple field modified gravity and localized energy in teleparallel framework. Gen. Relativ. Gravit., 8(47):1-14.

Bergmann PG, Thompson R, 1953. Spin and angular momentum in general relativity. Phys. Rev., 89: 400-407.

Einstein A, 1915. Preuss, Sitzungsber. Preuss. Akad. Wiss. Berlin, 47: 78 .

Halpern P, 2006. Energy of the Taub cosmological solution. Astrophys Space Sci., 306, 279-283.

Hehl FW, 1980. Proceeding of the 6h School of Cosmology and Gravitation on Spin, Torsion, Rotation and Supergravity. Erice, edited by P. Bergmann and V. de Sabbata, Plenum, New york, USA.

Landau LD, Liftshitz EM, 1951. The Classical Theory of Fields. Addison-Wesley Press, Reading, MA.

Maluf JW, 1994. Hamiltonian formulation of the teleparallel description of general relativity. J. Math. Phys., 35: 335-343.

Maluf JW, 1995. Localization of energy in general relativity. J. Math. Phys., 36: 4242-4247.

Maluf JW, Martins EF, Kneip A, 1996. Gravitational energy of rotating black holes. J. Math. Phys., 37: 6302-6310.

Maluf JW, da Rocha-Neto JF, 1999. General relativity on a null surface: Hamiltonian formulation in the teleparallel geometry. Gen. Rel. Grav., 31: 173-185.

Maluf JW, da Rocha-Neto JF, 2001. Hamiltonian formulation of general relativity in the teleparallel geometry. Phys. Rev. D, 64: 084014 . decreases in time and eventually it will vanish in both cases.

Maluf JW, da Rocha-Neto JF, Torbio TML, Castello-Branco KH, 2002. Energy and angular momentum of the gravitational field in the teleparallel geometry. Phys. Rev. D, 65: 124001.

Maluf JW, Ulhoa SC, Faria FF, da Rocha Neto JF, 2006. The angular momentum of the gravitational field and the Poincare group. Class. Quant. Grav., 23: 6245.

Maluf JW, Ulhoa SC, 2009. On the gravitational angular momentum of rotating sources. Gen. Rel. and Grav., 41(6): 1233-1247.

Maluf JW, 2013. The teleparallel equivalent of general relativity. Annalen der Physik, 525(5): 339-357.

Møller C., 1958. On the localization of the energy of a physical system in the general theory of relativity. Ann. Phys. (N.Y.), 4: $347-371$

Nashed GGL, 2008. Charged dilaton, energy, momentum and angular-momentum in teleparallel theory equivalent to general relativity. Eur. Phys. J. C, 54: 291-302.

Nashed GGL, 2010. Brane world black holes in teleparallel theory equivalent to general relativity and their Killing vectors, energy, momentum and angular momentum. Chin. Phys. B, 19: 020401

Papapetrou A, 1948. Einstein's theory of gravitation and flat space. Proc. R. Ir. Acad., 52: 11-23.

Qadir A, Sharif M, 1992. General formula for the momentum imparted to test particles in arbitrary spacetimes. Phys. Lett. A, 167: 331- 334 .

Rocha-Neto JF, Maluf JW, 2014. The angular momentum of planefronted gravitational waves in the teleparallel equivalent of general relativity. Gen. Rel. Grav., 46: 1667. 
Salti M, Acikgoz I, 2013. Black holes, wormholes and the Hamiltonian approach in the framework of teleparallel gravity. Physica Scripta 87(4): 045006.

Sharif M, Taj S, 2010. Energy Contents of Gravitational Waves in Teleparallel Gravity. Mod. Phys. Lett. A, 25: 221-232.

Sousa AA, Pareira RB, 2006. Angular momentum of the BTZ black hole in the teleparallel geometry. Prog. Theor. Phys., 114: $1179-1190$

Sousa AA, Moura JS, Pereira RB, 2010. Energy in an expanding universe in the teleparallel geometry. Braz. J. Phys., 40: 1-8.

Taub AH, 1951. Empty space-times admitting a three parameter group of motions. Ann. Math., 53: 472-490.
Tolman RC, 1934. Relativity, Thermodynamics and Cosmology. Oxford University Press, London, UK, 227 p.

Ulhoa SC, Rocha PMM, 2013. Neutron Stars in Teleparallel Gravity. Braz. J. Phys., 43(3): 162-171.

Weinberg S, 1972. Gravitation and Cosmlogy: Principles and Applications of General Theory of Relativity. Wiley, New York, USA, $165 \mathrm{p}$.

Weitzenböck R, 1923. Invarianten Theorie. Nordhoff, Groningen. 